\title{
Occupational stress andburnoutsyndrome in nurses whoact in emergency
}

\begin{abstract}
Objective: Which occasional factors to identify or the development of occupational stress and burnout syndrome in nurses working in the emergency.

Methods: This is a qualitative study using the method integrative literature review. The bibliography collected and selected for this study had the articles SciELO and LILACS, from 2012 to 2019 .

Results: Conducting the integrative literature review provided key interpretations and reflections for understanding the factors que lead to the development of occupational stress in nurses, bringing the the main results Following factors: overload and work, poor organization to work, lack of materials among other factors.
\end{abstract}

Conclusion: It concludes that it is necessary to create strategies, routines and norms, so that it is possible to offer psychological support to all health professionals.

Keywords: nursing, occupational stress, psychological exhaustion, emergency
Volume 4 Issue 2 - 2020

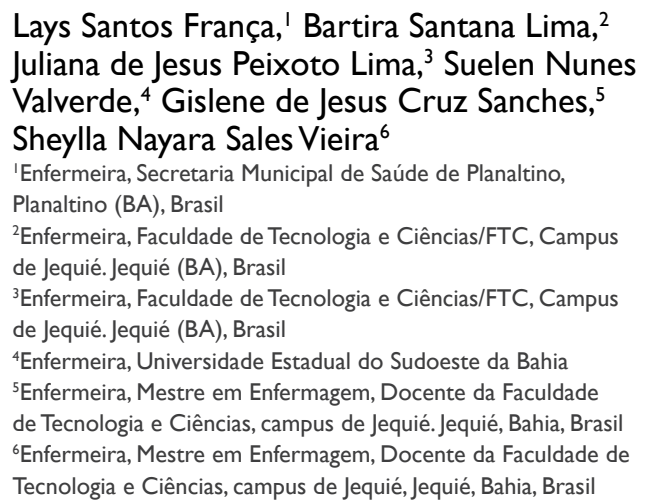

Correspondence: Lays France Santos, Nursing, Municipal Planaltino Health, Planaltino (BA), Brazil,

Email laysantos |20@gmail.com

Received: February 12, 2020 | Published: April 06, 2020

\section{Introduction}

The emergency service is the dynamic and complex sector as there are rapid changes in the clinical status of the patient therefore requires immediate attention, highly complex and inter-working, thus enabling the emergence of occupational stress among because nursing professionals the large flow of patients in this sector, which makes it high risk. Stress is known as a public health problem, in which the social dimension of the changes taking place in society causes an increase to its natural character. ${ }^{1}$ On the desktop, especially in hospital settings, but specifically in the emergency department, stress can have negative repercussions for both physical health as the subject of mental, depending on the length of stay, the nature and intensity of the relationships that are developed. ${ }^{2}$ Studies show that among all the stress factors existing in the profession can be considered as key: a decrease in the number of employees who are part of the nursing team that produces an increase in workload; the need for compliance with obligations in a short period; job dissatisfaction; lack of experience by supervisors; lack of communication and understanding on the part of the service supervision; and physical environment of the unit. ${ }^{3-5}$

It is noteworthy that currentlynursing professionals who work in this industry, experience at all times several factors that predispose to these problems, such as growing demand, increased accident numbers, lack of a structured network and the lack of beds, which can contribute to stress at work. It is very common place stress on professionals who work directly in emergencies, as they are the first to receive and house the patients, as well as every day these professionals experience difficult situations that most often require decision making and rapid assistance. ${ }^{6}$ It also includes the fact that the largest of nurses due to poor wage bill end up having to have more than a working relationship to achieve meet their needs, and their families, thus leading to an increased workload and a decrease in leisure time, social and family life. ${ }^{7}$

In this context, it requires professional full control by cooperating to the significant increase in levels of stressors and therefore a breakdown, also known as the Burnout syndrome, manifested by feelings of physical and mental exhaustion that may be associated with a feeling of frustration and failure nurse. ${ }^{7}$ Historically, according to the World Health Organization - WHO, Burnout syndrome, is a chronic syndrome resulting from stress in the workplace. It is considered an occupational syndrome, which may stem from feelings of exhaustion or physical and mental exhaustion; sense of self-deprecation, feelings of negativity or cynicism related to the work itself resulting in reduced professional efficacy. ${ }^{8}$

The inclusion of psychological counseling in order to relieve stress generated by routine work, and in turn, the establishment of favorable conditions requires the hospital management diversity of skills, respecting the specificities of each profession. This study is justified in seeking to understandthe effects of Burnout syndrome can generate as much for the professional who is in the front line of care and the patient receiving care that professional. In this sense, making it important to your research to determine the factors of prevention, which can be deployed in healthcare institutions to provide a safe working environment and consequently an effective care, generating an improvement in the quality of nursing care to patients. Thus, this study sought to answer the following research question: What are the conditions that favor the occupational stress of nurses in an emergency service? This question was based the whole construction of this study, basing the directions for bibliographic and interpretive 
research that became the basis for writing this article. Thus, this study aims to identify factors that cause the development of occupational stress and burnout in nurses working in the emergency.

\section{Methodology}

The theoretical and methodological approach chosen for achieving this paper included the qualitative approach with the method integrative review of the literature. This type of method allows synthesizing knowledge enabling incorporation of the applicability of the results of previous and significant studied in practice while providing the relevant research analysis, pointing certain knowledge gaps that can be filled with the production of new research and studies. In this sense, the referent method "consists in building a comprehensive literature review, contributing to discussions on methods and results of research, as well as reflections on the future studies". ${ }^{9}$ The process of gathering bibliographic data was held in February 2019, setting up a moment of reflection and study. Then sought to systematize data through fichamentos, abstracts, enumerations quotes, and personal stamp of observations necessary for the development of the article. The bibliography reviewed and selected for this study is site database SciELO (Cientifc Electronic Library Online), LILACS (Literature Latin American and Caribbean Health Sciences), which sought to scientific articles from the 2014 period to 2019 . The following detailed reading was taken and the printed articles proposed as literature. Careful and scientific nature reading, sought to recognize and understand data and information that are relevant to the theme, relating aspects and knowledge that were articulated in the proposed debate and present interpretive paths to this reality.

In this perspective, an integrative literature review provides the researcher a set of concepts, notes and reflections that may lead to new perspectives on the subject, identifying different ways of understanding the same and thus enabling answer the research problem. Propose a work in this type of methodology is to highlight the scientific dynamic and give visibility to what is discussing today on the subject. Thus, for the preparation of this article the following key words to search have been defined: Nurse, Occupational Stress, Exhaustion and psychological emergency. Therefore, after the intersection of descriptors combined with the Boolean AND, they found three hundred seventy-four articles. At first the articles were separated by year of publication, then the exclusion criteria were defined: Articles in a foreign language, repeated previous articles to the year 2014 and incomplete, and as inclusion criteria were used: Articles that are related to discussed topic; are in Portuguese, have been published or written during the period from 2013 to 2019. Then on the basis of inclusion and exclusion criteria were pre-selected seventeen articles, being used only seven of these. Because it is a literature review and not directly involved this research humans did not pass by the Research Ethics Committee, or presents ethical, as the Statement of Consent Form (ICF)

\section{Results and discussions}

The realization of integrative literature review provided an opportunity interpretations and reflections fundamental to the understanding of the factors that lead to the development of occupational stress in nurses in the care of patients in a hospital emergency room. Such reflections are characterized by being a relevant discussion to deepen the theme in the nursing field. The production of a literature review has the opportunity to produce interpretations and reflections on the theme chosen for research, in order to create a systematic set of information knowledge allowing understand the factors that cause occupational stress, as well as burnout in nurses working in the emergency room. In this paper, by analyzing all authors to build this study it was observed that the main triggering factors of burnout are high workload and the limited number of employees, factors that that lead to emotional exhaustion or physical these professionals..$^{5-7,10,11}$

Burnout syndrome is considered for many years as an important subject of research due to its high rate of incidence / prevalence and being assessed an occupational hazard. Several studies highlight the high risk of developing this syndrome among health professionals, especially among the nursing staff. ${ }^{2}$ Several studies have shown that female nurses working in the hospital emergency department are more likely to suffer burnout being justified by the fact that the double presence of women at home and at work, lower or higher pay demands at work. ${ }^{12}$ Regarding the main symptoms of the syndrome can find nervousness, physical and mental exhaustion, prostration, frequent headache, high blood pressure, muscle pain, gastrointestinal problems, changes in cárdicos rate, changes in appetite and mood, insomnia, difficulty concentrating, feelings of failure, incompetence and insecurity, isolation and constant negativity. Stress and lack of desire to get out of bed or home, when constant, may indicate the onset of disease. ${ }^{13}$

Therefore propose that the discussion on this subject is presented as relevant, given that the physical and mental health of nurses is characterized an important mechanism for quality care to patients admitted to the emergency.

Because it is in front of the line of care, the nursing staff is more provides to develop occupational stress, as well as burnout, so the profession is in fourth place in the ranking of the most demanding jobs in the public service, however there are few health facilities that provide a psychological support to these. When it concerns the nurse working in a very busy environment where patient demand is higher than the other sectors, there is various triggering factors for burnout syndrome as the workloads of high work in order to improve the financial return to the hospital, the large number of patients in the industry, poor physical infrastructure and material that exists in a large part of hospitals funded by Health-SUS System, among other factors that end up contributing to quality care is not offered increasing the patient's stress levels and low realization of this professional. ${ }^{6,14}$

This overload of work ends up generating, in addition to job stress and burnout, a family conflict, for professional ends up having his attention more work making ends leaving aside the family moments, missing important moments like growth of children. And above all leaving to care for themselves. ${ }^{15}$ For this reason that some studies indicate that it is necessary and very important to the implementation of strategies that allow nursing professionals the opportunity to voice their feelings of anxiety, dissatisfaction, insecurity and conflict, that are preventing its patient care. ${ }^{16}$ That being the role of the manager to provide its staff means to reduce occupational stress. Support and received help from co-workers and the hospital manager, also has a great ability to help reduce the stress and tension that occurs at work through interpersonal relationships between staff in the work environment. ${ }^{17}$ However we know that interpersonal relationships between the nursing staff within and outside the workplace are increasingly worn, causing the support and assistance do not exist in the workplace. Why it is necessary to create strategies, rules and routines, so you can provide psychological support to all health professionals, especially for nurses and their team, because it's no 
use to worry only about the quality of patient care without having a sense of how is the physical and mental health of nursing team professionals. ${ }^{18-20}$

\section{Conclusion}

When performing this study we can see that it is of great importance and it is important to study more and more about this subject. Even when it comes to theme that is found in various literatures is still high number of cases of professionals due to stress in the workplace end up presenting the burnout, however despite being a subject much discussed, there are few preventive measures, which also carries the high number of cases. With this study we can identify that work overload, the large number of patients, the environment with a poor physical structure, the lack of material, among others, are the factors that are directly related to the emergence of occupational stress and burnout among health professionals, especially among nurses and all the nursing staff who work directly in the emergency. We hope that this work, as well as the cited literature, serve as a subsidy to other studies on the subject matter, as well as warning serves for us to have a look out for the health of nursing professionals and not just worry about the care provided to the patient as if the professional is not with the physical and mental health in good condition the care provided to patients will be of poor quality.

\section{Acknowledgments}

None.

\section{Conflicts of interest}

The author declares there is no conflict of interest.

\section{Funding}

None.

\section{References}

1. Llapa AND-Rodriguez, JKA Oliveira, D Lopes Neto, et al. Occupational stress in nursing professionals. Rev enferm UERJ. 2018;26:e19404.

2. Portero de la Cruz S, Vaquero AM. Professional burnout, stress and job satisfaction of nursing staff at the university hospital. Rev Latino Am Nursing. 2015;23(3):543-552.

3. RTP Fernandes, Coelho MJ. emergency overcrowding: a new scenario for the care / nursing care. Rev electron Estacio Health. 2013;2(1):19-23.

4. Kolhs M, Olschowsky A, Barreta NL. In emergency and urgency Nursing: between pleasure and suffering. Rev Fund Care Online. 2017;9(2):42231.
5. Tan GS, Silveira PCR, Mininel VA, et al. Quality of life at work and occupational stress of nursing unit ready Customer. Text context Nurse. 2019;28:e20180298.

6. Andrioli AC, Frizon G, Ascari RA, et al. Stress causes of emergency nursing and emergency staff: a literature review. Magazine Uningá. 2016;47:58-64.

7. Bezerra CMB, Silva KKM, Costa JWS, et al. Prevalence of stress and burnout in nurses in hospital work in shifts. REME Rev Enferm Min. 2019;23:E-1232.

8. WHO. 11 $11^{\text {th }}$ International Classification of doenças- CID 11: QD85 occupational strain syndrome. 2019.

9. Smith C, Luiza Akiko Komura, Marina Peduzzi, et al. Integrative review: concepts and methods used in nursing. REEUSP. 2014;4 (2):335-344.

10. Baldonedo Monastery M, Almeida MCS, Baptista PCP, et al. Burnout syndrome in Brazilian and Spanish nursing workers. Rev Latino-Am Nursing. 2019;27:e3192.

11. Nogueira LS, Sousa RMC, Guedes ES, et al. Burnout and nursing work environment in public health Institutions. Rev Bras Nurse. 2018;71(2):336-342.

12. Oliveira BE, CH Gallasch, Silva Junior PPA, et al. Occupational stress and burnout in the emergency room nurses: the organization of work. Rev nurse UERJ Rio de Janeiro. 2017;25:e28842.

13. Brazil. Ministry of Health. Burnout syndrome: causes, symptoms, treatment, diagnosis and prevention. 2018

14. LA Almeida, Isadora Dantas de Souza Medeiros, Adriana Gonçalves de Barros, et al. Factors generators of burnout syndrome in health care professionals. Journal of Research: Care is Fundamental Online. 2016;8(3):4623-4628.

15. Freitas TLL, Foschera JA, Schneider V MECR. Burnout syndrome: implications conflict between work and family relationships. Barbaroi, Santa Cruz do Sul. 2018;(51):212-226.

16. MS Vasconcelos, Martino MMF. Predictors of burnout syndrome in intensive care unit nurses. Rev Gaúcha Enferm. 2017;38(4):e65354.

17. Ribeiro RP, Marziale MHP, Martins JT, et al. Occupational stress among health workers at a university hospital. Rev Gaúcha Enferm. 2018;39:e65127.

18. Cross SP. Related factors likely to suffer mental health problems in emergency professionals. Rev Latino-Am Nursing Ribeirão Preto. 2019;27:e3144.

19. Mosque KL, GPLA Gomes, Silva MJBF. The sight of the nurse / manager about the need to implement psychological support to professionals in the emergency mobile service. R Enferm Cent O min. 2014;4(1):1019-1028.

20. Mourao AL, Costa ACC, Silva EMM. Burnout syndrome in the context of nursing. Rev Bahian public health. 2017;41(1):131-143. 